\title{
Energy Decay of Damped Systems
}

\author{
Krešimir Veselićc*
}

\begin{abstract}
We present a new and simple bound for the exponential decay of second order systems using the spectral shift. This result is applied to finite matrices as well as to partial differential equations of Mathematical Physics. The type of the generated semigroup is shown to be bounded by the upper real part of the numerical range of the underlying quadratic operator pencil.
\end{abstract}

\section{Introduction and main estimate}

In this note we consider the abstract second order system

$$
M \ddot{x}+C \dot{x}+K x=0
$$

where $M, C, K$ are selfadjoint operators in a Hilbert space $\mathcal{X}$ with the scalar product $x^{*} y$ linear in the second and antilinear in the first variable. ${ }^{1}$ For simplicity, we assume that the operators $M, K, C$ are positive definite and that $M, C$ are bounded. The phase space formulation of (11) reads

$$
\begin{gathered}
\dot{y}=\mathcal{A} y, \quad \mathcal{A}=\left[\begin{array}{rr}
0 & K^{1 / 2} M^{-1 / 2} \\
-M^{-1 / 2} K^{1 / 2} & -M^{-1 / 2} C M^{-1 / 2}
\end{array}\right], \\
y=\left[\begin{array}{c}
K^{1 / 2} x \\
M^{1 / 2} \dot{x}
\end{array}\right],
\end{gathered}
$$

with the solution

$$
y=e^{\mathcal{A} t} y^{0} .
$$

Thus, the square of the norm equals twice the total energy of the system:

$$
\|y\|^{2}=\left\|K^{1 / 2} x\right\|^{2}+\dot{x}^{*} M \dot{x} .
$$

*Fernuniversität Hagen, Lehrgebiet Mathematische Physik, Postfach 940, D-58084 Hagen, Germany, e-mail: kresimir.veselic@fernuni-hagen.de.

${ }^{1}$ All other conventions and notations will be taken from $[5]$. 
The operator $\mathcal{A}$ is readily seen to be maximal dissipative on

$$
\mathcal{D}(\mathcal{A})=\mathcal{D}\left(K^{1 / 2}\right) \oplus M^{1 / 2} \mathcal{D}\left(K^{1 / 2}\right) .
$$

and thus the semigroup $e^{\mathcal{A} t}$ is contractive i.e. the energy of the system is a non-increasing function of $t .^{2}$ The resolvent of $\mathcal{A}$ is immediately seen to be given by

$$
(\mathcal{A}-\lambda I)^{-1}=\left[\begin{array}{cc}
-\frac{1}{\lambda}+\frac{1}{\lambda} K^{1 / 2} K(\lambda)^{-1} K^{1 / 2} & -K^{1 / 2} K(\lambda)^{-1} M^{1 / 2} \\
M^{1 / 2} K(\lambda)^{-1} K^{1 / 2} & -\lambda M^{1 / 2} K(\lambda)^{-1} M^{1 / 2}
\end{array}\right]
$$

with

$$
K(\lambda)=\lambda^{2} M+\lambda C+K
$$

at least for those $\lambda$ for which $K(\lambda)$ remains positive definite. This formula is rigorous, if $M, C, K$ are all bounded, otherwise the terms $M^{1 / 2} K(\lambda)^{-1} K^{1 / 2}$ and $K^{1 / 2} K(\lambda)^{-1} K^{1 / 2}$ have to be replaced by their closures; the latter are obviously everywhere defined and bounded.

Most existing works on the exponential decay estimate the infimum of all $\beta$ for which

$$
\left\|e^{A t}\right\| \leq C_{\beta} e^{\beta t} .
$$

The infimum value $\omega_{0}(A)$ of all possible $\beta$ in (6) (the type of the semigroup in the terminology of [5]) is often equal to the maximal real part of the spectrum of $\mathcal{A}$. In looking for this infimum usually little attention is paid to the constant $C_{\beta}$ which may tend to infinity as $\beta$ approaches the infimum (see e.g. [4], [2], 3] ). Since this constant plays a key role in controlling the finite-time behaviour of the system we are interested in a bound in which both $\beta$ and $C_{\beta}$ are tried to be made simply computable from the coefficients $M, C, K$. In fact, we obtain a family of estimates (6) for any $\beta$ from the interval $(\gamma, 0]$, where

$$
\gamma=\sup _{\substack{x \in \mathcal{D}\left(K^{1 / 2}\right) \\ x \neq 0}} \Re \frac{-x^{*} C x+\sqrt{\left(x^{*} C x\right)^{2}-4 x^{*} M x\left\|K^{1 / 2} x\right\|^{2}}}{2 x^{*} M x},
$$

while $C_{\beta}$ is an expression with similar ingredients. The set

$$
W(K)=\left\{\lambda \in C ; x^{*} K(\lambda) x=0, \text { for some unit } x\right\}
$$

is called the numerical range of the matrix pencil $K(\lambda)$ (cf. e.g [6]). Our result implies, in fact,

$$
\omega_{0}(\mathcal{A}) \leq \gamma=\sup \Re W(K) .
$$

\footnotetext{
${ }^{2}$ The reader primarily interested in finite dimensional applications may skip operatortheoretical details and just take $M, C, K$ as matrices. In this context 'positive' means 'positive semidefinite' and the expression $\left\|K^{1 / 2} x\right\|^{2}$ below may always be read as $x^{*} K x$.
} 
The bound for $\omega_{0}(\mathcal{A})$, obtained in [2] reads in our notations

$$
\gamma_{b}=\max \left\{-\inf _{x} \frac{x^{*} C x}{2 x^{*} M x},-\frac{1}{\sup _{x} \frac{x^{*} C x}{x^{*} K x}+2 \sqrt{\sup _{x} \frac{x^{*} K x}{x^{*} M x}}}\right\} .
$$

The values $\gamma$ and $\gamma_{b}$ are not easy to compare in general. For any underdamped system i.e. whenever the expression under the square root in (17) is uniformly negative, we obviously have $\gamma \leq \gamma_{b}$. For further comparisons see Sect. 2 below.

An estimate for $C_{\beta}$ was obtained in [3], Vol. I, Ch. 6. for the wave equation with distributed viscous damping. The bound obtained there is much less explicit than ours; this actually made impossible any comparison of the two.

As a by-product, we prove that $\omega_{0}(\mathcal{A})$ is equal to the supremum of the real part of the spectrum of $\mathcal{A}$ for "partly overdamped systems" i.e. for those for which $2 \gamma M+C$ is positive definite. Another case in which this equality is shown to hold are the so-called modally damped systems.

Our main tool will be the 'spectral shift substitution'

$$
x=e^{\mu t} z
$$

which gives rise to a new phase space representation, equivalent to the previous one. This yields a fairly simple total-energy decay estimate. Applications are made to both finite matrices and differential operators of Mathematical Physics. The obtained estimate is shown to be (asymptotically) attainable and therefore in some weak sense optimal. On the other hand, our estimate is void, if damping has a nontrivial nulspace. While there are such systems with no exponential decay at all, there are still many relevant cases whose decay is not covered by our theory. In Sec. 2 we provide illustrating examples.

The substitution $x=e^{\mu t} z$ yields

$$
\begin{gathered}
\dot{x}=\mu e^{\mu t} z+e^{\mu t} \dot{z} \\
\ddot{x}=\mu^{2} e^{\mu t} z+2 \mu e^{\mu t} \dot{z}+e^{\mu t} \ddot{z}
\end{gathered}
$$

and (11) reads

$$
M \ddot{z}+C(\mu) \dot{z}+K(\mu) z=0
$$

with

$$
C(\mu)=2 \mu M+C .
$$

As long as $C(\mu), K(\mu)$ stay positive definite this is equivalent to the phase space representation

$$
\dot{w}=\widehat{A} w, \quad \widehat{A}=\left[\begin{array}{rr}
0 & K(\mu)^{1 / 2} M^{-1 / 2} \\
-M^{-1 / 2} K(\mu)^{1 / 2} & -M^{-1 / 2} C M^{-1 / 2}
\end{array}\right],
$$




$$
w=\left[\begin{array}{r}
K(\mu)^{1 / 2} z \\
M^{1 / 2} \dot{z}
\end{array}\right],
$$

with the solution

$$
w=e^{\widehat{A} t} w^{0} .
$$

We now connect these two representations. We have

$$
\begin{gathered}
y_{1}=K^{1 / 2} x=e^{\mu t} K^{1 / 2} z=\mu e^{\mu t} K^{1 / 2} K(\mu)^{-1 / 2} w_{1}, \\
y_{2}=M^{1 / 2} \dot{x}=\mu e^{\mu t} M^{1 / 2} K(\mu)^{-1 / 2} w_{1}+e^{\mu t} w_{2} .
\end{gathered}
$$

Thus,

$$
\begin{gathered}
y=e^{\mu t} \mathcal{L}(\mu) w \\
\mathcal{L}(\mu)=\left[\begin{array}{rr}
K^{1 / 2} K(\mu)^{-1 / 2} & 0 \\
\mu M^{1 / 2} K(\mu)^{-1 / 2} & I
\end{array}\right] .
\end{gathered}
$$

This, together with the evolution equations for $y, w$ gives

$$
\mathcal{L}(\mu) \widehat{A}=(A-\mu I) \mathcal{L}(\mu) .
$$

This yields the decay estimate

$$
\left\|e^{A t}\right\| \leq\|\mathcal{L}(\mu)\|\left\|\mathcal{L}(\mu)^{-1}\right\| e^{\mu t} .
$$

The foregoing formal calculation is rigorous for finite matrices. Our general operator setting requires additional justifications of these steps. Also, we need a more explicit bound on the condition number which appears on the right hand side of (15). These two issues are the subject of the following. We set

$$
p_{ \pm}(x)=-\frac{x^{*} C x}{2 x^{*} M x} \pm \sqrt{D(x)}
$$

with $^{3}$

$$
D(x)=\left(\frac{x^{*} C x}{2 x^{*} M x}\right)^{2}-\frac{\left\|K^{1 / 2} x\right\|^{2}}{2 x^{*} M x}
$$

and

$$
\gamma=\sup _{\substack{x \in \mathcal{D}\left(K^{1 / 2}\right) \\ x \neq 0}} \Re p_{+}(x)
$$

Proposition 1 Let $M, C, K$ be as defined above. Then (i)

$$
\gamma \geq-\inf _{x} \frac{x^{*} C x}{2 x^{*} M x}
$$

moreover, $\gamma$ is the infimum of all $\mu$ for which both $K(\mu)$ and $2 \mu M+C$ are positive definite.

\footnotetext{
${ }^{3} \mathrm{By}$ convention, the square root of a negative number will have the positive imaginary part.
} 
Proof. The relation (18) is obvious. To prove the second assertion note that for any real $\mu$ the selfadjoint operator $K(\mu)$ is generated by the form

$$
h_{\mu}(x, y)=\mu^{2} x^{*} M y+\mu x^{*} C y+\left(K^{1 / 2} x\right)^{*} K^{1 / 2} y
$$

which is symmetric, bounded from below and closed on $\mathcal{D}\left(K^{1 / 2}\right)$. Take any $\mu>\gamma$. Then

$$
h_{\mu}(x, x)=x^{*} x\left(\mu-p_{-}(x)\right)\left(\mu-p_{+}(x)\right) \geq x^{*} x\left(\mu-\Re p_{+}(x)\right)^{2} \geq x^{*} x(\mu-\gamma)^{2}
$$

(note that $p_{-}(x) \leq p_{+}(x)$ whenever $D(x) \geq 0$ ). Thus, $K(\mu)$ is positive definite. By (18) $2 \mu M+C$ is positive definite also.

Conversely, suppose that $2 \gamma M+C$ is positive definite; there is a sequence $x_{n}$ of unit vectors such that

$$
\Re p_{+}\left(x_{n}\right)=-\frac{x_{n}^{*} C x_{n}}{2 x_{n}^{*} M x_{n}}+\Re \sqrt{D\left(x_{n}\right)} \rightarrow \gamma, \quad n \rightarrow \infty .
$$

Since $2 \gamma M+C$ is positive definite it follows

$$
\lim \inf _{n \rightarrow \infty} \Re \sqrt{D\left(x_{n}\right)}>0
$$

Thus, for $n$ sufficiently large $D\left(x_{n}\right)>0$, hence

$$
p_{+}\left(x_{n}\right)=\Re p_{+}\left(x_{n}\right) \rightarrow \gamma, \quad n \rightarrow \infty
$$

and $(K(\gamma)$ is positive semidefinite)

$$
h_{\mu}\left(x_{n}, x_{n}\right)=\left\|K(\gamma)^{1 / 2} x_{n}\right\|^{2}=\left(\gamma-p_{-}\left(x_{n}\right)\right)\left(\gamma-p_{+}\left(x_{n}\right)\right) \rightarrow 0
$$

since $p_{-}\left(x_{n}\right)$ is obviously bounded. Thus, $K(\gamma)$ has not a bounded inverse, and is not positive definite. Q.E.D.

Theorem 1 Let $M, C, K$ be as defined above. Assume that $\gamma<\mu \leq 0$ holds. Then (i) the operator $\mathcal{L}(\mu)$ together with its inverse is bounded and everywhere defined. (ii) Both operators leave the subspace $\mathcal{D}(A)$ invariant and 14) holds. (iii) The estimate (6) holds with

$$
\beta=\mu, \quad C_{\beta}=\|\mathcal{L}(\mu)\|\left\|\mathcal{L}(\mu)^{-1}\right\| .
$$

(iv) The type of the semigroup is bounded by

$$
\omega_{0}(\mathcal{A}) \leq \gamma
$$


Proof. Since $\gamma$ is negative then for $-\gamma<\mu \leq 0$ both $K(\mu)$ and $C(\mu)$ remain positive definite. The boundedness of $M, C$ implies (cf. [5] )

$$
\mathcal{D}\left(K(\mu)^{1 / 2}\right)=\mathcal{D}\left(K^{1 / 2}\right), \quad \text { and } \quad \mathcal{D}(K(\mu))=\mathcal{D}(K), \quad 0>\mu>\gamma,
$$

so both (13) and its inverse

$$
\mathcal{L}(\mu)^{-1}=\left[\begin{array}{cc}
K(\mu)^{1 / 2} K^{-1 / 2} & 0 \\
-\mu M^{1 / 2} K^{-1 / 2} & I
\end{array}\right]
$$

are everywhere defined and bounded (a straightforward calculation shows that the one is the inverse of the other). This also shows that $\mathcal{D}(A)=\mathcal{D}(\widehat{A})$.

Take a vector from $\mathcal{D}(A)$, that is, $x \in \mathcal{D}\left(K^{1 / 2}\right), y \in M^{1 / 2} \mathcal{D}\left(K^{1 / 2}\right)$ and set

$$
\mathcal{L}(\mu)\left[\begin{array}{l}
x \\
y
\end{array}\right]=\left[\begin{array}{l}
u \\
v
\end{array}\right] .
$$

Here $x=K^{1 / 2} K(\mu)^{-1 / 2} x$ is from $\mathcal{D}\left(K^{1 / 2}\right)$ because $K(\mu)^{-1 / 2} x \in \mathcal{D}(K(\mu)=$ $\mathcal{D}(K)$. Also $v=\mu M^{1 / 2} K(\mu)^{-1 / 2} x+y$ is from $M^{1 / 2} \mathcal{D}\left(K^{1 / 2}\right)$ because $y$ is such and $K(\mu)^{-1 / 2} x \in \mathcal{D}(K)$. Thus, $\mathcal{L}(\mu)$ leaves $\mathcal{D}(A)$ invariant. The proof for $\mathcal{L}(\mu)^{-1}$ is similar. Now the relation (14) can be directly verified on any vector from $\mathcal{D}(A)$. The estimate (19) is now obvious. Q.E.D.

\section{Corollary 1 If}

$$
\gamma>-\inf _{x} \frac{x^{*} C x}{2 x^{*} M x}
$$

then

$$
\gamma=\omega_{0}(\mathcal{A})=\sup \sigma(\mathcal{A})
$$

Proof. Under our assumption, as in the proof of Proposition 1, it follows that $K(\gamma)$ is not boundedly invertible while $K(\lambda)$ is positive definite for any $\lambda>\gamma$. Now, the 2,2-block in the resolvent matrix in (4) gets unbounded for $\lambda=\gamma$. Thus, all $\lambda$ with $\Re \lambda>\gamma$ belong to the resolvent set of $\mathcal{A}$ whereas $\gamma$ belongs to its spectrum, hence $\gamma=\sup \Re \sigma(\mathcal{A})$. From Theorem 1 it follows $\omega_{0}(\mathcal{A}) \leq \gamma$ while $\omega_{0}(\mathcal{A}) \geq \sup \sigma(\mathcal{A})$ is a general fact. So, the assertion follows. Q.E.D.

The systems covered by the corollary above may be called 'partially overdamped' in a sense similar to that introduced in [1].

We next give some bounds for the condition number appearing in (19). Note that both $\mathcal{L}(\mu)$ and $\mathcal{L}(\mu)^{-1}$ are of the type

$$
\mathcal{L}=\left[\begin{array}{ll}
A & 0 \\
B & I
\end{array}\right]
$$


Lemma 1 Let $A, B$ be bounded operators and Then $\|\mathcal{L}\|$ from (22) is bounded by any of the numbers

$$
\sqrt{1+\left\|A^{*} A+B^{*} B\right\|}, \quad \max \{\|A\|, 1+\|B\|\}, \quad \max \{\|A\|+\|B\|, 1\} .
$$

Proof. We have

$$
\mathcal{L}^{*} \mathcal{L}=\left[\begin{array}{cc}
A^{*} A+B^{*} B & B^{*} \\
B & I
\end{array}\right], \quad \mathcal{L} \mathcal{L}^{*}=\left[\begin{array}{cc}
A A^{*} & A B^{*} \\
B A^{*} & B B^{*}+I
\end{array}\right] .
$$

If $B=0$ the assertion is trivial. If $B \neq 0$ the number $a=\|\mathcal{L}\|^{2}=\left\|\mathcal{L} \mathcal{L}^{*}\right\|=$ $\left\|\mathcal{L}^{*} \mathcal{L}\right\|>1$ belongs to the spectrum of $\mathcal{L}^{*} \mathcal{L}$ and there exist sequances $x_{n}, y_{n}$ with $\left\|x_{n}\right\|^{2}+\left\|y_{n}\right\|^{2}=1$ and

$$
\begin{aligned}
\left(A^{*} A+B^{*} B\right) x_{n}+B^{*} y_{n}-a x_{n} & \rightarrow 0 \\
B x_{n}+y_{n}-a y_{n} & \rightarrow 0
\end{aligned}
$$

for $n \rightarrow 0$. Hence

$$
\left(A^{*} A+B^{*} B+\frac{B^{*} B}{a-1}-a\right) x_{n} \rightarrow 0
$$

By (25) the sequence $x_{n}$ does not converge to 0 and without loss of generality we may assume that in (24) $\left\|x_{n}\right\|=1$ holds. Then

$$
a^{2}-a\left(\left(A^{*} A+B^{*} B+I\right) x_{n}, x_{n}\right)+\left(A^{*} A x_{n}, x_{n}\right) \rightarrow 0 .
$$

Hence

$$
\begin{gathered}
a \leq \sup _{\|x\|=1} \frac{\left(\left(A^{*} A+B^{*} B+I\right) x, x\right)+\sqrt{\left.\left(A^{*} A+B^{*} B+I\right) x, x\right)^{2}-4(x, x)\left(A^{*} A x, x\right)}}{2} \\
\leq \sup _{\|x\|=1}\left(\left(A^{*} A+B^{*} B+I\right) x, x\right) .
\end{gathered}
$$

The other two bounds in (23) are obvious. Q.E.D.

Remark 1 (i) Ours is, in fact a family of bounds depending on the parameter $\mu$ from the interval $(\gamma, 0]$. An optimal bound would be obtained as the infimum over all of them.

(ii) By continuity, the bound (19) remains valid even for $\mu=\gamma$, if $K(\gamma)$ is positive definite. In this case we have $\omega_{0}(\mathcal{A})<\gamma$ i.e. or bound is not optimal. Indeed, for some $\mu<\gamma$ the operator $K(\mu)$ will still be positive definite and $\widehat{A}$ will still generate a uniformly bounded semigroup ([5], Ch. IX, Th. 2.1) and (14), (15) will still be valid. 
(iii) Our main estimate does not contain the norms of the operators $M, C$ or their inverses and indeed it would certainly hold in much more singular cases, but then some additional regularity conditions would be needed e.g. the boundedness of the operators $C^{1 / 2} K^{-1 / 2}, C^{-1 / 2} M^{1 / 2}$ and the like thus requiring more technical proofs. The same is valid, if we would admit more general damping operator $C$ by merely asking it to be accretive.

(iv) The expression (17) for $\gamma$ is neat but not easily computable, even in the case of finite matrices. As suggested by Proposition 1 a simple viable method to determine $\gamma$ numerically would run as follows

- Find $\gamma_{0}=-\inf \frac{x^{*} C x}{2 x^{*} M x}$ by finding the lowest eigenvalue of the matrix pencil $2 \lambda M-C$.

- If $\gamma_{0} \approx 0$ halt, no bound available.

- If $\gamma_{0}<0$ and $K\left(\gamma_{0}\right)$ is positive definite then $\gamma=\gamma_{0}$.

- If $\gamma_{0}<0$ and $K\left(\gamma_{0}\right)$ is not positive definite then seek $\gamma$ by bisection in the interval $\left(\gamma_{0}, 0\right]$.

\section{Examples}

Our first example is the one-dimensional system with the two-dimensional phase space matrix

$$
A=\left[\begin{array}{rr}
0 & k \\
-k & -d
\end{array}\right], \quad k>0, \quad d>0
$$

A straightforward, if a bit tedious, computation gives

$$
e^{A t}=\left[\begin{array}{cc}
\cos \delta t+d(\sin \delta t) /(2 \delta) & k(\sin \delta t) / \delta \\
-k(\sin \delta t) / \delta & \cos \delta t-d(\sin \delta t) /(2 \delta)
\end{array}\right] e^{-d t / 2}
$$

with

$$
\delta=\sqrt{4 k^{2}-d^{2}} / 2 .
$$

The above formula is valid for $\delta$ both positive and negative (in the latter case there is a real expression by means of hyperbolic functions) whereas the formula for $4 k^{2}-d^{2}=0$ is obtained taking the limit $k \rightarrow d / 2$, thus reading

$$
e^{A t}=\left[\begin{array}{cc}
1+d t / 2 & d t / 2 \\
-d t / 2 & 1-d t / 2
\end{array}\right] e^{-d t / 2}
$$

Here

$$
\mathcal{L}(\mu)=\left[\begin{array}{cc}
\frac{k}{\sqrt{\mu^{2}+\mu d+k^{2}}} & 0 \\
\frac{\mu}{\sqrt{\mu^{2}+\mu d+k^{2}}} & 1
\end{array}\right]
$$


and our Theorem above yields

$$
\gamma=\Re \frac{-d+\sqrt{d^{2}-4 k^{2}}}{2}
$$

Here the right hand side is the largest real part of the spectrum and therefore the inequality (20) is, in fact, an equality i.e. our estimate for $\omega_{0}(\mathcal{A})$ is sharp for all possible values of $k, d$. The bound from [2] reads (in our notations)

$$
\gamma_{b}=\max \left\{-\frac{k^{2}}{d+2 k},-\frac{d}{2}\right\} .
$$

As a straightforward calculation shows we have here $\gamma_{b}=\gamma$ for $d \leq(\sqrt{3}-1) k$ and $\gamma_{b}>\gamma$ for $d>(\sqrt{3}-1) k$.

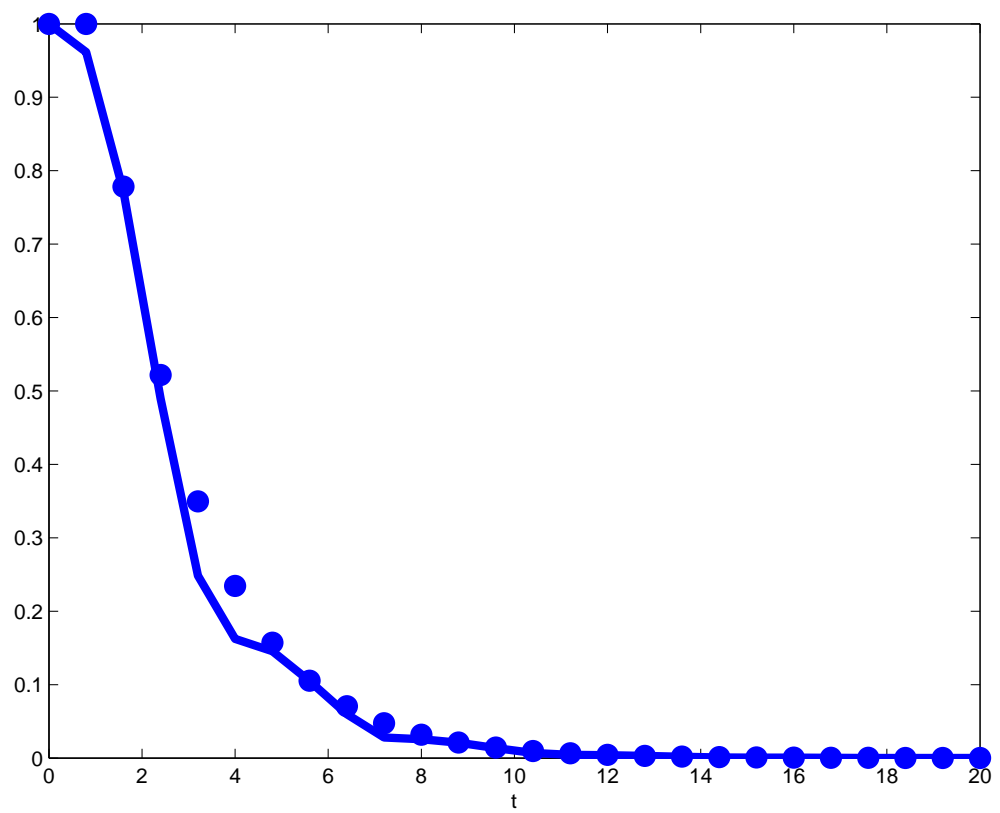

Figure 1: $k / d=1$

On Figs. 1, 2 we display our bound (dotted line) with the true norm (solid line) as functions of $t$ (our bound is obtained by taking the minimum over four equidistant values of $\mu$ ). We see that our constant $C_{\beta}$ gets pessimistic for 


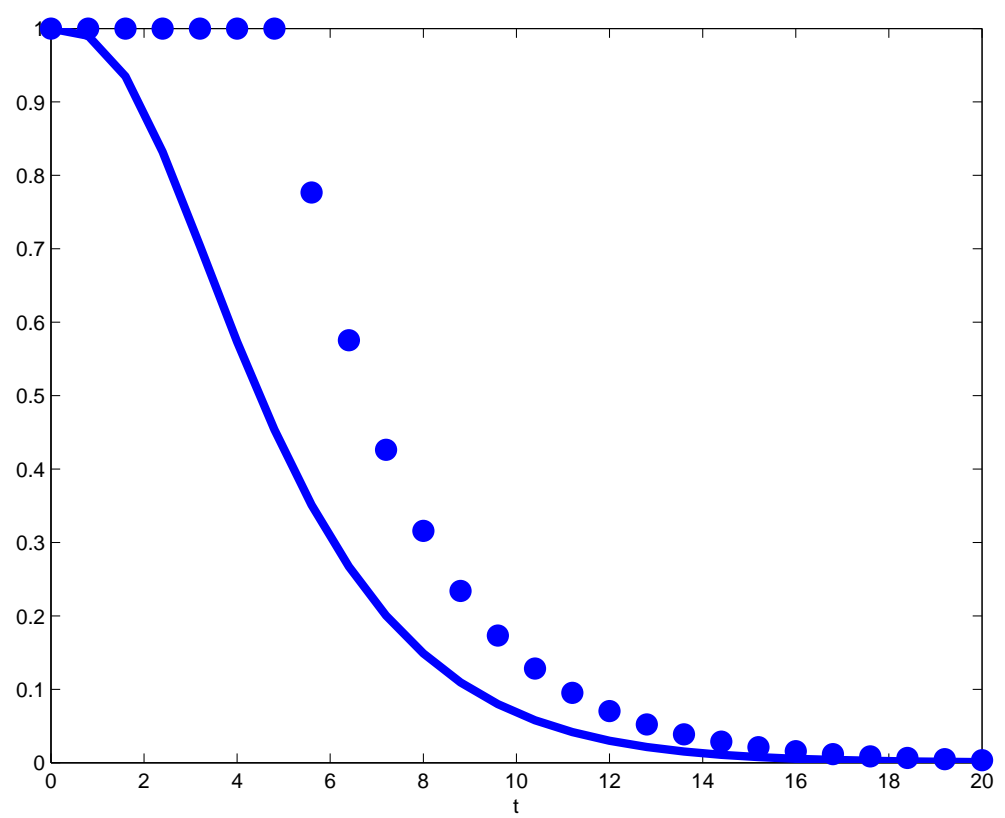

Figure 2: $\quad k / d=0.5$

small values of $k / d$. On the other hand, for $k / d \rightarrow \infty$ we may take $\mu=d / 2$, $\mathcal{L}(\mu) \rightarrow I$ and (19) reads asymptotically

$$
\left\|e^{A t}\right\| \leq e^{d t / 2}
$$

whereas the right hand of (29) behaves as

$$
\left[\begin{array}{cc}
\cos k t & \sin k t \\
-\sin k t & \cos k t
\end{array}\right] e^{-d t / 2} .
$$

Thus, or bound (19) is asymptotically sharp.

Any modally damped system i.e. a system in which $M, K, C$ satisfy the relation

$$
C M^{-1} K=K M^{-1} M
$$

is easily seen to be unitarily equivalent to an orthogonal sum of matrices of type (28), a bound is obtained as the maximum over all of them. The type $\omega_{0}(\mathcal{A})$ is the maximum of the values in $(\underline{31}) \cdot{ }^{4}$ In particular, $\omega_{0}(\mathcal{A})$ is equal to $\Re \sigma(\mathcal{A})$.

As a second example take the wave equation in a bounded domain $\Omega \subset R^{n}$

$$
w_{t t}(x, t)+c(x) w_{t}(x, t)+\Delta w(x, t)=0,
$$

\footnotetext{
${ }^{4}$ This remark is literally true if the system has discrete spectrum (e.g., if $K$ has a compact inverse). Otherwise we would have direct integrals.
} 
with the boundary condition

$$
w(x, t)=0 \quad x \in \partial \Omega .
$$

The function $c$ is supposed to have finite positive minimum and maximum.

We will estimate e.g. the second of the bounds in (23). Taking first

$$
A=K^{1 / 2} K(\mu)^{-1 / 2}, \quad B=\mu M^{1 / 2} K(\mu)^{-1 / 2}
$$

in $\mathcal{X}=L_{2}(R)$ we have

$$
\|A\|^{2}=\frac{1}{1+\inf _{u} \frac{u^{*}\left(\mu^{2}+\mu c(\cdot)\right) u}{\|\nabla u\|^{2}}}=\frac{1}{1+\lambda_{1}},
$$

where $\lambda_{1}$ is the lowest eigenvalue $\lambda$ of the boundary value problem

$$
\left(\mu^{2}+\mu c(x)\right) u=-\lambda \Delta u,\left.\quad u\right|_{\partial \Omega}=0 .
$$

Since under our assumptions $\mu^{2}+\mu c(x)$ is negative definite we have

$$
\lambda_{1}<0, \quad 1+\lambda_{1}>0 \text {. }
$$

Furthermore

$$
\begin{gathered}
\|B\|^{2}=\sup _{u} \frac{\mu^{2} u^{*} u}{u^{*}\left(\mu^{2}+\mu c(\cdot)-\Delta\right) u}=\frac{1}{\mu^{2}+\inf _{u} \frac{u^{*}(\mu c(\cdot)-\Delta) u}{u^{*} u}} \\
=\frac{1}{\mu^{2}+\lambda_{2}},
\end{gathered}
$$

where $\lambda_{2}$ is the lowest eigenvalue $\lambda$ of the boundary value problem

$$
(\mu c(x)-\Delta) u=\lambda u,\left.\quad u\right|_{\partial \Omega}=0 .
$$

Taking next

$$
A=K(\mu)^{1 / 2} K^{-1 / 2}, \quad B=-\mu M^{1 / 2} K^{-1 / 2},
$$

we have $\|A\| \leq 1$ and

$$
\|B\|^{2}=\frac{1}{\lambda_{3}}
$$

where $\lambda_{3}$ is the lowest eigenvalue $\lambda$ of the boundary value problem

$$
\Delta u=-\lambda \mu u,\left.\quad u\right|_{\partial \Omega}=0 .
$$

Altogether

$$
C_{\mu}=\|\mathcal{L}(\mu)\|\left\|\mathcal{L}^{-1}(\mu)\right\| \leq \max \left\{\frac{1}{\sqrt{1+\lambda_{1}}}, 1+\frac{1}{\sqrt{\mu^{2}+\lambda_{2}}}\right\}\left(1+\frac{1}{\sqrt{\lambda_{3}}}\right),
$$

where $\lambda_{1}, \lambda_{2}, \lambda_{3}$ are obtained above. Thus, our bound for $C_{\mu}$ is obtained from the extremal eigenvalues of some selfadjoint elliptic boundary value problems involving $M, C, K$. If $c(x)$ is constant then all these boundary value problems reduce to $-\Delta u=\lambda u$. This system is also modally damped, so the corresponding estimates are applicable here, too. 


\section{References}

[1] E. M. Barston, A minimax principle for nonoverdamped systems, Internat. J. Engrg. Sci. 12 (1974), 413-421.

[2] A. Bátkai, K.-J. Engel, Exponential decay of $2 \times 2$ operator matrix semigroups, to appear in Journal of Computational Analysis and Applications.

[3] G. Chen, J. Zhou, Vibration and Damping in Distributed Systems, CRC Press Boca Raton 1993.

[4] S. Cox, E. Zuazua, The rate at which energy decays in a damped string, Commun. Part. Diff. Eq. 19 (1994) 213-243.

[5] T. Kato, Perturbation Theory for Linear Operators, Springer Berlin 1966.

[6] A. S. Markus, L. Rodman, Some results on numerical ranges and factorizations of matrix polynomials, Linear and Multilinear Algebra 42 (1997), 169-185. 


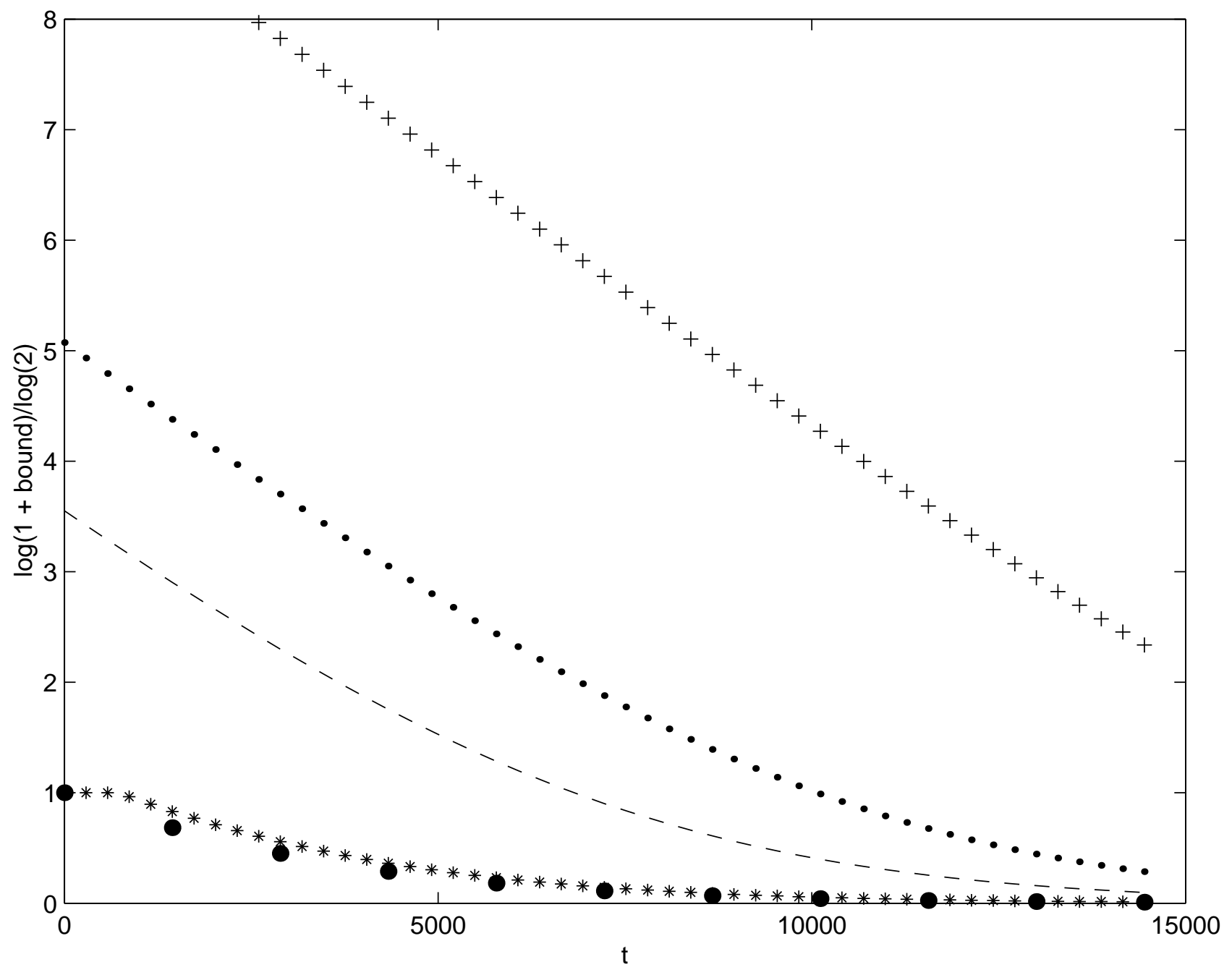

